\title{
Evidence for two $\Lambda(1405)$ resonance states
}

\author{
E. Oset ${ }^{a}$, V. K. Magas ${ }^{b}$ and A. Ramos ${ }^{b}$
}

November 23, 2018

\author{
${ }^{a}$ Departmento de Física Teórica and IFIC, Centro Mixto Universidad de Valencia-CSIC, \\ Institutos de Investigación de Paterna, Aptd. 22085, 46071 Valencia, Spain \\ ${ }^{b}$ Departament d'Estructura i Constituents de la Matéria, \\ Universitat de Barcelona, Diagonal 647, 08028 Barcelona, Spain
}

\begin{abstract}
The $K^{-} p \rightarrow \pi^{0} \pi^{0} \Sigma^{0}$ reaction is studied within a chiral unitary model. The distribution of $\pi^{0} \Sigma^{0}$ states forming the $\Lambda(1405)$ shows, in agreement with a recent experiment, a peak at $1420 \mathrm{MeV}$ and a relatively narrow width of $\Gamma=38 \mathrm{MeV}$. We use these data in combination with those of the $\pi^{-} p \rightarrow K^{0} \pi \Sigma$ reaction and elements of chiral unitary theory to prove that there are two $\Lambda(1405)$ states instead of one as so far assumed.
\end{abstract}

The history of the $\Lambda(1405)$ as a dynamical resonance generated from the interaction of meson baryon components in coupled channels has experienced a boost within the context of unitary extensions of chiral perturbation theory $(U \chi P T)$ [1, 2, 3, 4, 5], where the lowest order chiral Lagrangian and unitarity in coupled channels generates the $\Lambda(1405)$ and leads to good agreement with the $K^{-} p$ reactions. The surprise, however, came with the realization that there are two poles in the neighborhood of the $\Lambda(1405)$ both contributing to the final experimental invariant mass distribution [3, 4, 5, 6, 7, 8, 9]. The properties of these two states are quite different, one has a mass around $1390 \mathrm{MeV}$, a large width of about $130 \mathrm{MeV}$ 
and couples mostly to $\pi \Sigma$, while the second one has a mass around $1425 \mathrm{MeV}$, a narrow width of about $30 \mathrm{MeV}$ and couples mostly to $\bar{K} N$. The two states are populated with different weights in different reactions and, hence, their superposition can lead to different distribution shapes. Since the $\Lambda(1405)$ resonance is always seen from the invariant mass of its only strong decay channel, the $\pi \Sigma$, hopes to see the second pole are tied to having a reaction where the $\Lambda(1405)$ is formed from the $\bar{K} N$ channel. This is accomplished by the recently measured reaction $K^{-} p \rightarrow \pi^{0} \pi^{0} \Sigma^{0}$ [10] which allows us to test already the two-pole nature of the $\Lambda(1405)$.

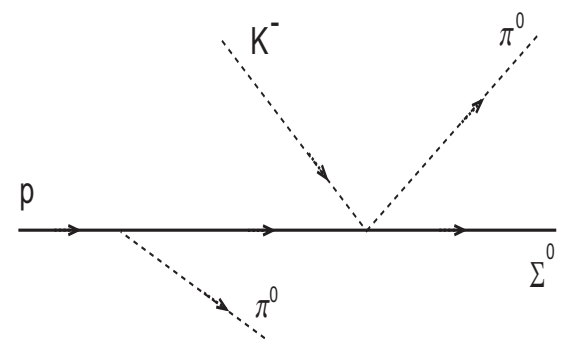

Figure 1: Nucleon pole term for the $K^{-} p \rightarrow \pi^{0} \pi^{0} \Sigma$ reaction.

Our model for the reaction $K^{-} p \rightarrow \pi^{0} \pi^{0} \Sigma^{0}$ in the energy region of $p_{K^{-}}=514$ to 750 $\mathrm{MeV} / \mathrm{c}$, as in the experiment [10], considers those mechanisms in which a $\pi^{0}$ loses the necessary energy to allow the remaining $\pi^{0} \Sigma^{0}$ pair to be on top of the $\Lambda(1405)$ resonance. The first of such mechanisms is given by the diagram of Fig. 1] Other mechanisms that involve the meson meson interaction and BBMMM vertices were found negligible in the detailed study of [11].
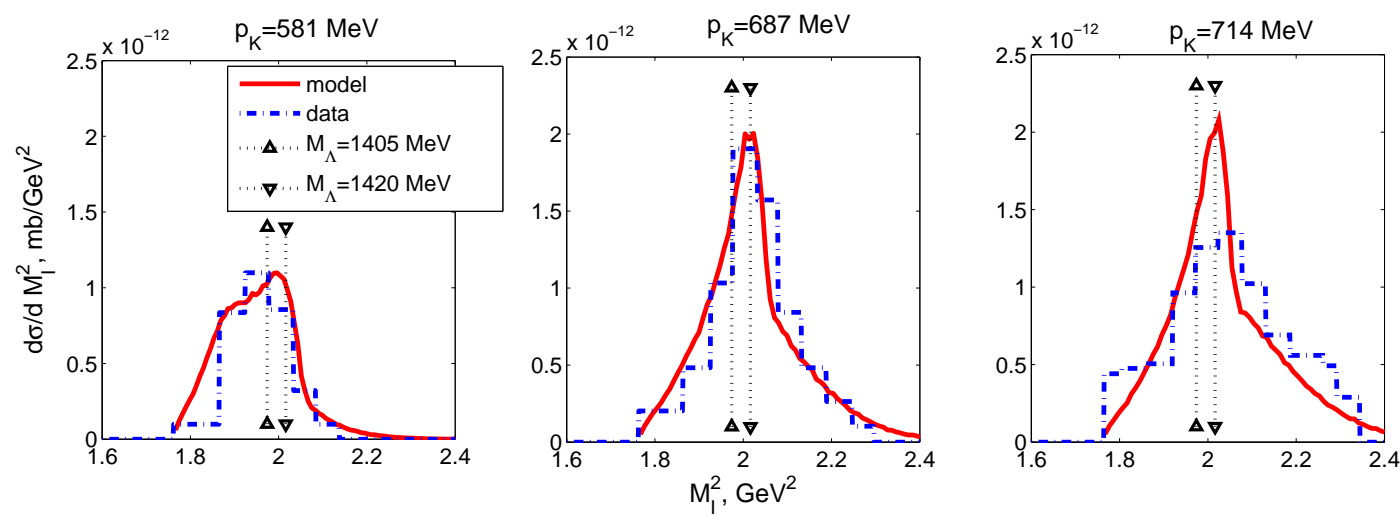

Figure 2: The $\left(\pi^{0} \Sigma^{0}\right)$ invariant mass distribution for three different initial kaon momenta. 
The amplitude for the process $K^{-} p \rightarrow \pi^{0} \pi^{0} \Sigma^{0}$, considering the diagram of Fig. 1 with a pseudovector $\pi N N$ coupling (and keeping up to $1 / M_{N}$ terms) is given by:

$$
\begin{aligned}
& -i t_{K^{-} p \rightarrow \pi^{0} \pi^{0} \Sigma^{0}}=-\frac{D+F}{2 f} \vec{\sigma}\left[\vec{q}^{\prime}\left(1+\frac{q^{0 \prime}}{2 M_{N}}\right)+\frac{q^{0 \prime}}{M_{N}} \vec{k}\right] \times \\
& \frac{M_{N}}{E_{N}\left(\vec{k}+\vec{q}^{\prime}\right)} \frac{1}{E_{N}(\vec{k})-q^{\prime 0}-E_{N}\left(\vec{k}+\vec{q}^{\prime}\right)} t_{K^{-} p \rightarrow \pi^{0} \Sigma^{0}},
\end{aligned}
$$

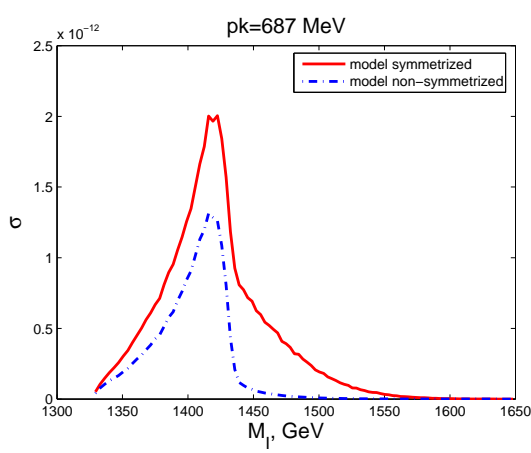

Figure 3: Theoretical $\left(\pi^{0} \Sigma^{0}\right)$ invariant mass distribution for an initial kaon lab momenta of 687 $\mathrm{MeV}$. The non-symmetrized distribution also contains the factor $1 / 2$ in the cross section.

where $q$ r refers to the momentum of the first outgoing pion and $k$ is the momentum of the kaon (see Fig. 11). The second pion, from the decay of ther $\Lambda(1405)$, is assumed to have a momentum $q_{f}$.

The indistinguishability of the two emitted pions requires the implementation of symmetrization. This is achieved by summing two amplitudes evaluated with the two pion momenta exchanged, $q_{f} \leftrightarrow q$. In addition, a factor of $1 / 2$ for indistinguishable particles is also included in the total cross section.

Our calculations show that the process is largely dominated by the nucleon pole term shown in Fig. 1. As a consequence, the $\Lambda(1405)$ thus obtained comes mainly from the $K^{-} p \rightarrow \pi^{0} \Sigma^{0}$ amplitude which, as mentioned above, gives the largest possible weight to the second (narrower) state.

In Fig. 2 our results for the invariant mass distribution for three different energies of the incoming $K^{-}$are compared to the experimental data. Symmetrization of the amplitudes produces a sizable amount of background. At a kaon laboratory momentum of $p_{K}=581$ 


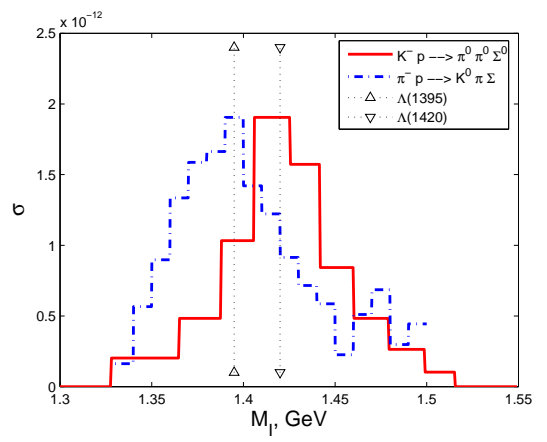

Figure 4: Two experimental shapes of $\Lambda(1405)$ resonance. See text for more details.

$\mathrm{MeV} / \mathrm{c}$ this background distorts the $\Lambda(1405)$ shape producing cross section in the lower part of $M_{I}$, while at $p_{K}=714 \mathrm{MeV} / \mathrm{c}$ the strength of this background is shifted toward the higher $M_{I}$ region. An ideal situation is found for momenta around $687 \mathrm{MeV} / \mathrm{c}$, where the background sits below the $\Lambda(1405)$ peak distorting its shape minimally. The peak of the resonance shows up at $M_{I}^{2}=2.02 \mathrm{GeV}^{2}$ which corresponds to $M_{I}=1420 \mathrm{MeV}$, larger than the nominal $\Lambda(1405)$, and in agreement with the predictions of Ref. [6] for the location of the peak when the process is dominated by the $t_{\bar{K} N \rightarrow \pi \Sigma}$ amplitude. The apparent width from experiment is about $40-45 \mathrm{MeV}$, but a precise determination would require to remove the background mostly coming from the "wrong" $\pi^{0} \Sigma^{0}$ couples due to the indistinguishability of the two pions. A theoretical analysis permits extracting the pure resonant part by not symmetrizing the amplitude. This is plotted in Fig. 3 as a function of $M_{I}$. The width of the resonant part is $\Gamma=38 \mathrm{MeV}$, which is smaller than the nominal $\Lambda(1405)$ width of $50 \pm 2 \mathrm{MeV}$ [13], obtained from the average of several experiments, and much narrower than the apparent width of about $60 \mathrm{MeV}$ that one sees in the $\pi^{-} p \rightarrow K^{0} \pi \Sigma$ experiment [14, which also produces a distribution peaked at $1395 \mathrm{MeV}$. In order to illustrate the difference between the $\Lambda(1405)$ resonance seen in this latter reaction and in the present one, the two experimental distributions are compared in Fig. 4. We recall that the shape of the $\Lambda(1405)$ in the $\pi^{-} p \rightarrow K^{0} \pi \Sigma$ reaction was shown in Ref. [12] to be largely built from the $\pi \Sigma \rightarrow \pi \Sigma$ amplitude, which is dominated by the wider lower energy state.

The invariant mass distributions shown here are not normalized, as in experiment. But we can also compare our absolute values of the total cross sections with those in Ref. [10]. As shown in Fig. 5. our results are in excellent agreement with the data, in particular for the 


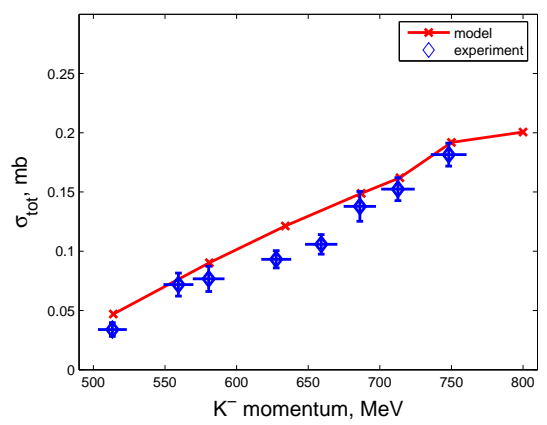

Figure 5: Total cross section for the reaction $K^{-} p \rightarrow \pi^{0} \pi^{0} \Sigma^{0}$. Experimental data are taken from Ref. [10].

three kaon momentum values whose corresponding invariant mass distributions have been displayed in Fig. 2

In summary, we have shown, by means of a realistic model, that the $K^{-} p \rightarrow \pi^{0} \pi^{0} \Sigma^{0}$ reaction is particularly suited to study the features of the second pole of the $\Lambda(1405)$ resonance, since it is largely dominated by a mechanism in which a $\pi^{0}$ is emitted prior to the $K^{-} p \rightarrow \pi^{0} \Sigma^{0}$ amplitude, which is the one giving the largest weight to the second narrower state at higher energy. Our model has proved to be accurate in reproducing both the invariant mass distributions and integrated cross sections seen in a recent experiment [10. The study of the present reaction, complemental to the one of Ref. [12] for the $\pi^{-} p \rightarrow K^{0} \pi \Sigma$ reaction, has shown that the quite different shapes of the $\Lambda(1405)$ resonance seen in these experiments can be interpreted in favour of the existence of two poles with the corresponding states having the characteristics predicted by the chiral theoretical calculations. Besides demonstrating once more the great predictive power of the chiral unitary theories, this combined study of the two reactions gives the first clear evidence of the two-pole nature of the $\Lambda(1405)$.

Acknowledgments: This work is partly supported by DGICYT contracts BFM200201868, BFM2003-00856, the Generalitat de Catalunya contract SGR2001-64, and the E.U. EURIDICE network contract HPRN-CT-2002-00311. This research is part of the EU Integrated Infrastructure Initiative Hadron Physics Project under contract number RII3-CT2004-506078. 


\section{References}

[1] N. Kaiser, P. B. Siegel and W. Weise, Phys. Lett. B 362 (1995) 23

[2] E. Oset and A. Ramos, Nucl. Phys. A 635 (1998) 99 arXiv:nucl-th/9711022.

[3] J. A. Oller and U. G. Meissner, Phys. Lett. B 500 (2001) 263 arXiv:hep-ph/0011146.

[4] D. Jido, A. Hosaka, J. C. Nacher, E. Oset and A. Ramos, Phys. Rev. C 66 (2002) 025203 arXiv:hep-ph/0203248.

[5] C. Garcia-Recio, J. Nieves, E. Ruiz Arriola and M. J. Vicente Vacas, Phys. Rev. D 67 (2003) 076009 arXiv:hep-ph/0210311.

[6] D. Jido, J. A. Oller, E. Oset, A. Ramos and U. G. Meissner, Nucl. Phys. A 725 (2003) 181 arXiv:nucl-th/0303062.

[7] C. Garcia-Recio, M. F. M. Lutz and J. Nieves, Phys. Lett. B 582 (2004) 49 arXiv:nucl-th/0305100.

[8] T. Hyodo, S. I. Nam, D. Jido and A. Hosaka, Phys. Rev. C 68 (2003) 018201 arXiv:nucl-th/0212026.

[9] S. I. Nam, H. C. Kim, T. Hyodo, D. Jido and A. Hosaka, arXiv:hep-ph/0309017.

[10] S. Prakhov et al. [Crystall Ball Collaboration], Phys. Rev. C 70 (2004) 034605.

[11] V. K. Magas, E. Oset and A. Ramos, Phys. Rev. Lett. 95 (2005) 052301 arXiv:hep-ph/0503043.

[12] T. Hyodo, A. Hosaka, E. Oset, A. Ramos and M. J. Vicente Vacas, Phys. Rev. C 68 (2003) 065203 arXiv:nucl-th/0307005.

[13] K. Hagiwara et al. [Particle Data Group], Phys. Rev. D 66, 010001 (2002).

[14] D. W. Thomas, A. Engler, H. E. Fisk, and R. W. Kraemer, Nucl. Phys. B 56, 15 (1973). 